\title{
Utilizing Fission Technology to Enable Rapid and Affordable Access to any Point in the Solar System
}

\author{
Mike Houts, Joe Bonometti, Jeff Morton, Ivana Hrbud, Leo Bitteker, Melissa Van \\ Dyke, Tom Godfroy, Kevin Pedersen, Chris Dobson, Bruce Patton, James Martin, \\ Suman Chakrabarti
}

\author{
NASA MSFC. TD40. Marshall Space Flight Center, Alabama, 35812 \\ michael.houts@mstc.nasa.gov/(256) 544-7143/Fax: (256)544-5926
}

\begin{abstract}
Fission technology can enable rapid, affordable access to any point in the solar system. Potential fission-based transportation options include bimodal nuclear thermal rockets, high specific energy propulsion systems, and pulsed fission propulsion systems. In-space propellant re-supply enhances the effective performance of all systems, but requires significant infrastructure development. Safe, timely, affordable utilization of firstgeneration space fission propulsion systems will enable the development of more advanced systems. First generation systems can build on over 45 years of US and international space fission system technology development to minimize cost.
\end{abstract}

\section{INTRODUCTION}

Fission technology can enable rapid, affordable access to any point in the solar system. Advanced concepts (i.e. the "Medusa" concept (Solem, 1993) and vapor or droplet core fission systems driving high-efficiency thrusters (Anghaie, 1999)) could reduce trip time to Mars, Jupiter, and beyond by an order of magnitude compared to today's systems. In the mid-term, bimodal nuclear thermal rockets with liquid oxygen afterburners (LANTR) could reduce earth-lunar transit time to 24 hours, enable affordable six-month transits to Mars, and explore much of the inner solar system utilizing in-situ propellant re-supply (Borowski, 1999). In-space propellant re-supply could greatly enhance the effective performance of all propulsion systems.

Compared to other advanced propulsion options, fission systems are conceptually quite simple. All that is required is for the right materials to be placed in the right geometry (no extreme temperatures or pressures required) and the system will operate. In addition, the fuel for fission systems (highly enriched uranium) is virtually non-radioactive, containing 0.064 curies $/ \mathrm{kg}$. This compares quite favorably to radioisotope systems (Pu-238 contains 17,000 curies $/ \mathrm{kg}$ ) and D-T fusion systems (tritium contains $10,000,000$ curies $/ \mathrm{kg}$ ). At launch, a typical space fission propulsion system would contain an order of magnitude less onboard radioactivity than Mars Pathfinder's Sojourner Rover. The primary safety issue with fission systems is avoiding accidental criticality - addressing this issue through proper system design is quite straightforward.

The potential of space fission systems is illustrated in Figure 1. As shown in the Figure, the energy density in fission systems is seven orders of magnitude greater than that of the best chemical systems. Put another way, completely fissioning a piece of uranium the size of a coke can would yield two orders of magnitude more energy than burning all of the chemical fuel contained in the space shuttle main tank. If properly harnessed, the energy density in fissile fuel far exceeds that required enabling rapid access to any point in the solar system. Additionally, the technology readiness level (TRL) of space fission systems is much higher than that of nuclear fusion, matter annihilation, and hot isomeric transition. Fission systems are the nearest-term option for high efficiency, high thrust in-space propulsion. 


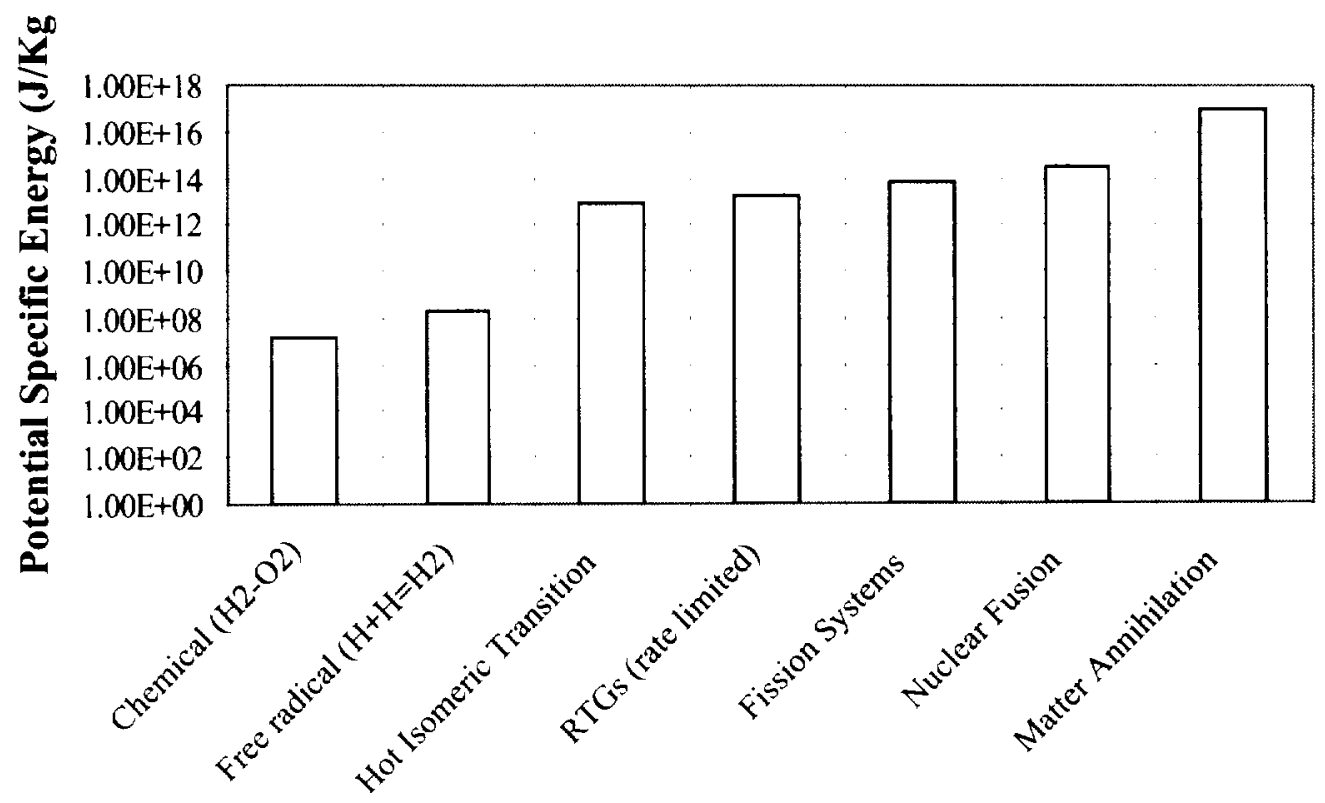

Candidate Propellant Energy Sources

FIGURE 1. Energy density of candidate propellant energy sources.

\section{FIRST GENERATION SPACE FISSION SYSTEMS}

Despite the relative simplicity and tremendous potential of space fission systems, the development and utilization of these systems has proven elusive. The first use of fission technology in space occurred 3 April 1965 with the US launch of the SNAP-10A reactor. There have been no additional US uses of space fission systems. While space fission systems were used extensively by the former Soviet Union, their application was limited to earth-orbital missions. Early space fission systems must be safely and affordably utilized if we are to reap the benefits of advanced space fission systems.

Table 1 gives a partial list of major US space fission programs that have failed to result in flight of a system. There are a variety of reasons why these programs failed to result in a flight. The fact that so many programs have failed indicates that a significantly different approach must be taken if future programs are to succeed.

The government, universities, industries, and utilities have utilized terrestrial fission systems for over 50 years. In addition, technology development directly related to space fission systems has been ongoing for over 40 years. The next generation fission system should capitalize on this experience. Nuclear testing can be one of the most expensive and time-consuming aspects of space fission system development. If a system can be designed to operate within established fuel burnup and component radiation damage limits, the requirement for nuclear testing can be minimized. Designing the system such that resistance heaters can be used to closely simulate heat from fission will also facilitate development and allow extensive testing of the actual flight unit.

Additional innovative approaches will have to be used to ensure that the next space fission system development program results in system utilization. Safety must be the primary focus of the program, but cost and schedule must also be significant drivers. System performance must be adequate, but the desire to make performance more than adequate should not be allowed to drive system cost and schedule. The next generation space fission system must be safe, simple, and as inexpensive to develop and utilize as possible.

One option for a first generation fission propulsion system is the First Generation Least Expensive Approach to Fission (FIGLEAF) system. A high temperature FIGLEAF module is currently on test, and full core FIGLEAF 
testing is scheduled to begin in FY 2000. Figure 2 is a picture of the FIGLEAF module operating at $1750 \mathrm{~K}$. Figure 3 is a picture of the FIGLEAF module operating at $1750 \mathrm{~K}$ with heat pipe simultaneously operating at $1450 \mathrm{~K}$. Additional details on the FIGLEAF are presented in (Van Dyke, 2000).

TABLE 1. Partial lists of major US Space Fission Programs that have failed to result in Flight of a System.

\begin{tabular}{|c|c|c|}
\hline $\begin{array}{l}\text { Solid-Core Nuclear Rocket } \\
\text { Program } \\
\text { Medium-Power Reactor } \\
\text { Experiment (MPRE) } \\
\text { Thermionic Tech. Program } \\
\text { (1963-1973) } \\
\text { Space Nuclear Thermal Rocket } \\
\text { Program } \\
\text { SP-100 }\end{array}$ & $\begin{array}{ll} & \text { SNAP-50/SPUR } \\
\text { - } & \text { High-Temperature Gas- } \\
& \text { Cooled Electric Power } \\
& \text { Reactor (710 Reactor) } \\
\text { - } & \text { SPAR / SP-100 } \\
\text { - } & \text { Flight Topaz } \\
\text { - } & \text { DOE } 40 \mathrm{k}[\mathrm{W} \text { e Thermionic } \\
& \text { Reactor Prog }\end{array}$ & $\begin{array}{l}\text { - } \\
\text { Cooled Reactor } \\
\text { - } \quad \text { Advanced Space Nuclear Power } \\
\text { Program (SPR) } \\
\text { - } \quad \text { Multi-Megawatt Program } \\
\text { - } \quad \text { Thermionic Fuel Element Veri. } \\
\text { Pro. } \\
\text { - } \quad \text { Air Force Bimodal Study }\end{array}$ \\
\hline
\end{tabular}

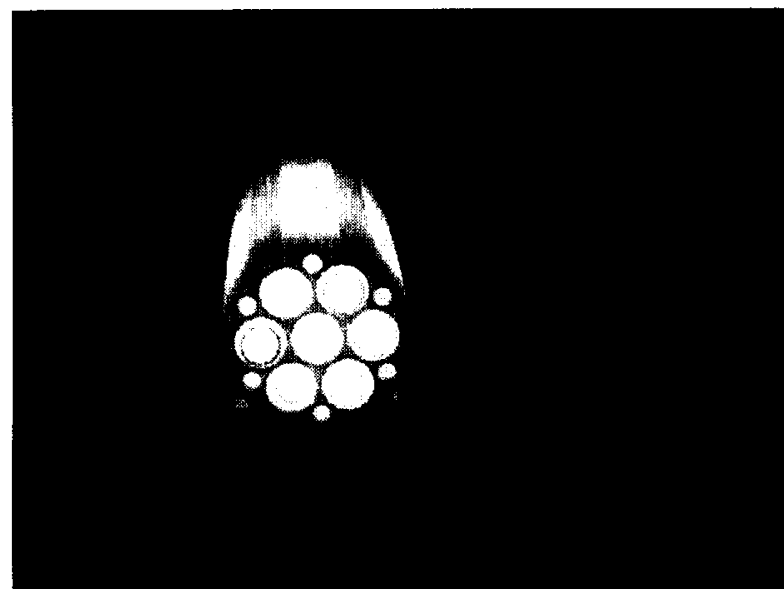

FIGURE 2. Portion of FIGLEAF Module at $1750 \mathrm{~K}$.

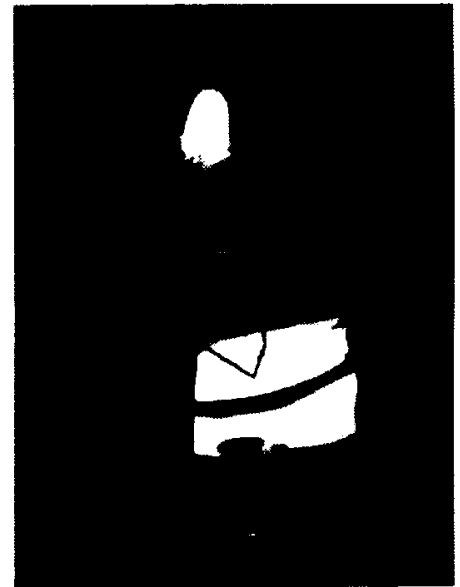

FIGURE 3. FIGLEAF Module.

\section{PULSED FISSION (OR FISSION/FUSION) PROPULSION SYSTEMS}

Pulsed propulsion systems have been under consideration since the late 1940's. In a pulsed propulsion system. fission or fission/fusion pulses that release between $10^{13}$ and $10^{15}$ Joules of energy are used to propel a vehicle. The primary difficulty with pulsed propulsion systems is coupling the pulse to the vehicle without exceeding limits on acceleration. If adequate coupling schemes are devised. effective specific impulses exceeding 50,000 seconds with vehicle thrust-to-weight ratios exceeding 1.0 are feasible. Nearer-term systems would be more likely to have specific impulses on the order of $4000 \mathrm{~s}$, still nearly an order of magnitude greater than the best chemical propulsion systems (Dyson, 1999).

In the energy range of interest the equivalent specific impulse of pulsed propulsion systems increases with the magnitude of the pulse. The primary obstacle to the utilization of large pulses is devising a method for spreading the acceleration out over a period of several seconds. Large pulses could deliver total impulses on the order of $10^{8} \mathrm{~kg}$ $\mathrm{m} / \mathrm{s}$ or more. Assuming a $200 \mathrm{MT}$ vehicle and a maximum acceleration limit of $250 \mathrm{~m} / \mathrm{s}^{2}$ thus requires that the impulse be delivered to the spacecraft over a minimum of two seconds. Another concern is the cost of the pulse unit. Because cost is not strongly dependent on pulse unit size, the use of large pulses may also result in less expensive missions. A $10^{14} \mathrm{~J}$ pulse requires the fissioning of roughly $1.25 \mathrm{~kg}$ of uranium. Future research related to pulsed propulsion systems should focus on methods for utilizing large pulses, on the order of $10^{14} \mathrm{~J}$ or higher. Systems utilizing large pulses could enable rapid access to any point in the solar system. 
Most previous work related to pulsed fission propulsion systems focused on earth-to-orbit systems. This focus drove vehicle designs to those capable of utilizing a rapid series of pulses, on the order of one pulse per second. A system requiring several minutes to reconfigure between pulses may be acceptable for in-space transfer applications. Dealing with variability in pulse sizes and the occasional failure of a pulse unit may also be simplified for in-space transfer applications.

\section{HIGH SPECIFIC ENERGY FISSION PROPULSION SYSTEMS}

This specific energy of fissile fuel is $8 \times 10^{13} \mathrm{~J} / \mathrm{kg}$. For systems requiring a year of operation at full thrust without refueling, the minimum theoretical specific mass is thus $4 \times 10^{-4} \mathrm{~kg} / \mathrm{kW}$. In an actual system, structure, heat removal, energy conversion, waste heat rejection, radiation shielding, and other subsystems will significantly increase specific mass. However, it may still be possible to devise high efficiency (Isp $>3000 \mathrm{~s}$ ) fission propulsion systems with a specific mass in the 0.1 to $1.0 \mathrm{~kg} / \mathrm{kW}_{\text {propellant }}$ range. These systems would enable rapid access to any point in the solar system.

Initial research on these systems could involve non-nuclear simulations of vapor or droplet core fission reactors. Advanced energy conversion subsystems including MHD energy conversion and high-temperature Brayton cycles could be investigated. Flowing $\mathrm{UF}_{4}$ (or other fuel-form) loops could be constructed (using natural or depleted uranium) to validate thermal hydraulic predictions and investigate high temperature materials compatibility.

\section{IN-SPACE PROPELLANT RE-SUPPLY}

The performance of any space propulsion system can in theory be enhanced with in-space propellant re-supply. Propellant re-supply increases the effective specific impulse of a given propulsion system compared to the same propulsion system without propellant re-supply, in some scenarios by nearly an order of magnitude. Several challenges must be overcome before in-space propellant re-supply can be utilized.

1. A source of propellant must be available at a desired location outside of a large gravity well. In a few instances the propellant source may already be at the desired location, otherwise it must be moved there.

2. A method for collecting and processing the propellant must be devised.

3. A method for storing the propellant until needed must be devised.

4. A method for effectively utilizing the propellant must be devised.

Perhaps the best potentially available propellant source is water. Water can both be electrolyzed and used in chemical propulsion systems or used directly in nuclear steam rockets (Zuppero, 1999). Unfortunately, there are no known in-space reservoirs of relatively pure water in the inner solar system. Numerous individuals have proposed bringing icy bodies from the outer solar system into the inner solar system - some of those proposals are discussed in Fogg, 1995. The discovery of the Kuiper Belt in 1992 further strengthened arguments for utilizing icy bodies from the outer solar system. It is now believed that there are over 30 billion Kuiper Belt Objects, the majority of them with a radius less than $1 \mathrm{~km}$ (Weissman, 1999). Centaurs (believed to originate in the Kuiper Belt) are also a potential source of water in the outer solar system. One Centaur, Chiron, has a radius of $150 \mathrm{~km}$ and an orbit that crosses that of Saturn.

The difficulty associated with moving propellant into a desired orbit can be thought of in terms of the required propulsive delta- $\mathrm{V}$. A very significant advantage of obtaining water from the outer solar system is that the required propulsive delta-V can be quite small, on the order of a few hundred meters per second (Fogg, 1995). The remainder of the required velocity change can be accomplished via gravity assist maneuvers. Imparting a velocity change of a few hundred meters per second to a large, icy object could be accomplished with a relatively near-term nuclear thermal rocket. For example, suppose a $500 \mathrm{~m} / \mathrm{s}$ velocity change is to be imparted on a 1 billion-kg icy object. If a nuclear steam rocket with an exhaust exit velocity of $1500 \mathrm{~m} / \mathrm{s}$ is used (attainable with stainless steel or superalloy clad fission systems) then a total of $2.84 \times 10^{8} \mathrm{~kg}$ of steam would need to be exhausted. Assuming that the reactor provides all of the kinetic energy for the steam, and then adding $25 \%$ to the energy requirement for thermal losses, results in a requirement that $5 \mathrm{~kg}$ of uranium be fissioned. Because of the relatively low core temperatures required by the steam rocket, a stainless-steel clad $\mathrm{UO}_{2}$ core should be viable. The propulsive delta- $\mathrm{V}$ could be imparted over a period of 50 days given a $100 \mathrm{MW}$ core, and for reasonable fuel loadings, established fuel 
burnup limits would not be exceeded. There would; however, still be significant challenges to developing the steam rocket, including propellant acquisition, impurity limits, and engine control.

One scenario for obtaining water ice in the inner solar system could thus be to heat a Kuiper Belt Object, Centaur, or other icy body (in-situ) and evaporate steam from the body. The steam would then be condensed inside a membrane, and the resulting ice balloon placed in the desired inner solar system orbit through a series of propulsive maneuvers and gravity assists. Although extremely challenging from an engineering standpoint. the physics required for obtaining water in this fashion is known.

Once water is in the desired orbit, it can either be used directly in steam rockets (Zuppero, 1999) or electrolyzed for use in chemical engines. If orbits are properly chosen and in-space propellant re-supply is used, fast planetary transfers can be accomplished using currently available rocket technology. For example, calculations (Kos, 1999) show that properly placed propellant re-supply stations can enable missions to Mars with one-way transit times of less than 70 days. These missions can be performed by any high thrust system. independent of the system's specific impulse and without the requirement for an aerobrake. Very robust, reusable vehicles could be utilized. Vehicles with propellant mass fractions of less than $50 \%$ would not be out of the question. Without in-space propellant resupply, a specific impulse on the order of $10,000 \mathrm{~s}$ would be required to perform an equivalent mission. With inspace propellant re-supply, engines with a specific impulse of $400 \mathrm{~s}$ (or less) could still accomplish the 70 day EarthMars transit.

While high specific impulse is not required for performing the missions, the use of high specific impulse systems (such as nuclear thermal rockets) reduces the required number of propellant re-supply stations. Calculations (Kos. 1999) also show that missions to the asteroid belt are feasible with 5 month transit times and missions to Jupiter are feasible with 13 month travel times, again using any high-thrust propulsion system.

A schematic for performing orbital changes via in-space propellant re-supply is shown in Figure 4. In the scenario shown, a Mars-bound spacecraft would be assembled in orbit, then fueled using propellant obtained from space. The spacecraft would then propel itself to earth escape, obtain propellant, propel itself into orbit 1, obtain propellant, propel itself into orbit 2, obtain propellant, then propel into a slightly hyperbolic trajectory towards Mars. Upon reaching Mars, a similar procedure would be used to slow down. All of the outbound propulsion would be performed near earth in as short of time period as possible (preferably a few hours). All propulsion required to rendezvous with Mars would likewise be performed in a short period of time.

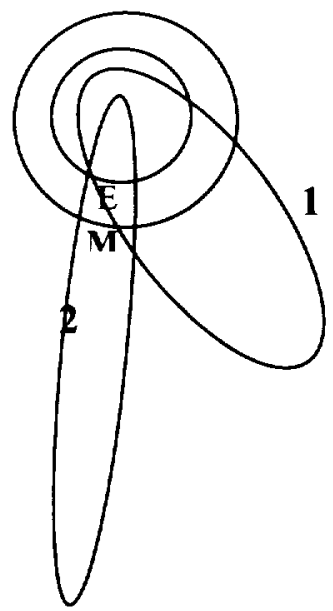

FIGURE 4. Schematic for orbit changing via propellant re-supply.

There are numerous challenges with the in-space propellant re-supply scenario. First, propellant re-supply stations must be in resonance orbits to allow periodic alignment of the re-supply stations. For example, in Figure 4 all of the re-supply stations must be near point "E" before the fast transit can be accomplished. Second, highly-automated spacecraft would be required to obtain the propellant, direct it into the correct orbit, and then maintain the correct 
orbit. Third, the window for performing the fast transit might last only a few days at each alignment, and a great number of re-supplies could be required by low performance propulsion systems. Fourth, rapid transfer of propellant from the re-supply station to the spacecraft could be difficult.

The primary advantage of using in-space propellant re-supply is that while logistically complex, all of the required physics is known. Once an in-space propellant re-supply infrastructure was in place, existing LOX/hydrogen engines would have the equivalent of an order of magnitude greater specific impulse for certain missions. Solidcore nuclear thermal rockets would have similarly improved effective performance.

\section{RECOMMENDATIONS FOR FUTURE RESEARCH}

Research should continue on a first generation fission propellant energy source. The focus of this research should be on demonstrating that fission propulsion systems can be developed and utilized in a safe, timely, and affordable fashion. Research on pulsed propulsion systems should focus on methods for utilizing large ( $1 \times 10^{14} \mathrm{~J}$ or greater) pulses. Research on high specific energy systems can be focused in a variety of areas. but should lead to systems capable of providing on the order of a kilowatt of power into the propellant for every kilogram of system mass. Research related to in-situ propellant re-supply can also be focused in a variety of areas, but should lead to the capability to place propellant re-supply stations where they are most needed for a given mission. Research needs related to the LANTR system are detailed elsewhere (Borowski, 1999).

\section{ACKNOWLEDGMENTS}

Unless otherwise referenced, the research reported in this paper was funded by and performed at NASA's Marshall Space Flight Center.

\section{REFERENCES}

Anghaie, Samim, Personal Communication, Innovative Nuclear Space Power and Propulsion Institute (INSPI), 1999.

Borowski, S.K., Dudzinski, L.A., and McGuire, M.L. "Bimodal NTR and LANTR Propulsion for Human Missions to Mars/Phobos" in Space Nuclear Power and Propulsion, edited by Mohamed S. El-Genk, AIP Conf 458 , American Institute of Physics, New York, 1999, pp. 1261-1268.

Dyson, F., Personal Communication, Princeton, NJ 1999.

Fogg, M.J. Terraforming: Engineering Planetary Environments, Society of Automotive Engineers, Inc. Warrendale, PA 15096-0001, 1995.

Kos, L., Personal Communication, Huntsville, AL 1999.

Solem, J.C. "Medusa: Nuclear Explosive Propulsion for Interplanetary Travel," Journal of the British Interplanetary Society, Vol. 46, pp. 21-26, 1993.

Vandyke, Melissa "Results of the Propellant Energy Source Module (PESM) Testing at the Marshall Space Flight Center: Non-Nuclear Testing of a Fission System" to be published in Space Nuclear Power and Propulsion, edited by Mohamed S. El-Genk, American Institute of Physics, New York, 2000, within these procedings.

Weissman, P.R., McFadden, L.A., and Johnson, T.V. Encyclopedia of the Solar System, Academic Press, San Diego, CA, 1999, pp. 557-583.

Zuppero, A., Larson, T.K., Schnitzler, B.G., Werner, J.E., Rice, J.W., Hill, T.J., Richins, W.D., and Parlier, L. "Origin of How Steam Rockets can Reduce Space Transport Cost by Orders of Magnitude" in Space Nuclear Power and Propulsion, edited by Mohamed S. El-Genk, AIP Conf 458, American Institute of Physics, New York, 1999, pp. 1211-1216. 